\title{
Simplexes and their applications - a short survey
}

\author{
István Szalkai and Balázs Szalkai
}




\title{
SIMPLEXES AND THEIR APPLICATIONS - A SHORT SURVEY
}

\author{
BALÁZS SZALKAI AND ISTVÁN SZALKAI
}

Received February 21, 2012

\begin{abstract}
We investigate different types of simplexes (linear algebraic, affine, geometric), solve some extremal problems and state general conjectures concerning these notions in discrete geometry and matroid theory, finally present some applications in chemistry and physics.

Connections to homogeneous system of linear equations are also mentioned.
\end{abstract}

2000 Mathematics Subject Classification: 05D05; 05A05; 05B35; 15A03; 15A06; 51A45

Keywords: stoichiometry, minimal reaction, linear algebra, simplex,matroid, hypergraph

\section{DeFinitions}

Since there are many different notions of simplexes in use in mathematics, first we have to present the definitions we need.

Definition 1. (Algebraic simplexes) In the $n$-dimensional linear (Euclidean) space $\mathbb{R}^{n}$ any set of vectors $\delta \subset \mathbb{R}^{n}$ is called a (linear) algebraic simplex iff $\delta$ is minimal linear dependent, i.e. $\delta$ is linearly dependent but each proper subset $\mathcal{T} \varsubsetneqq \delta$ is independent.

Remark 1 . The set $\delta=\{\underline{0}\}$ fulfills the previous definition, so it may be called (the only) 1-element simplex. Though this extremal case is not interesting in applications, but we need it in Remark 2.

The corresponding notion in matroids is called a circuit. Clearly linear algebraic simplexes are just matroidal circuits in special matroids.

Definition 2. (Geometric simplexes) Any set of $n+1$ points in $\mathbb{R}^{n}$ in general position is called a geometric simplex .

Describing affine simplexes runs by induction on the number of points. The definition might be easier if we first assume that one of the points is located at the origin, the other cases come after by translations.

\footnotetext{
* The second author express his many thanks to the Fields Institute (Toronto, Canada) for granting and inviting him to the Workshop on Optimization, September 26-29, 2011, for which occasion this survey was prepared.
} 
Definition 3. (Affine simplexes) Any finite set $\delta=\left\{\underline{s}_{1}, \underline{s}_{2}, \ldots, \underline{s}_{k}\right\} \subset \mathbb{R}^{n}$ is an affine simplex if and only if $k \geq 3$ and

o) in the case $k=3$ the points $\left\{\underline{s}_{1}, \underline{s}_{2}, \underline{s}_{3}\right\}$ must be colinear,

i) in the case $\underline{s}_{1}=\underline{0}$ and $k \geq 4$ we require the vectors

$$
\delta^{-}:=\left\{\underline{s}_{2}, \underline{s}_{3}, \ldots, \underline{s}_{k}\right\}
$$

to be linearly dependent, and no proper subset of (the original) 8 is an affine simplex, ii) in the general case $k \geq 4$ the set of vectors

$$
\mathcal{S}^{-}:=\left\{\underline{s}_{2}-\underline{s}_{1}, \underline{s}_{3}-\underline{s}_{1}, \ldots, \underline{s}_{k}-\underline{s}_{1}\right\}
$$

is required to be linearly dependent, but $\delta$ is not allowed to contain any affine simplex as a proper subset.

Cases $n=3$ and $n=4$ can be formulated in easier way.

Example 1. (i) A set of points $\delta \subset \mathbb{R}^{2}$ is an affine

$\triangleright \quad 3$-element simplex iff $\delta$ is three colinear points,

$\triangleright \quad 4$-element simplex iff 8 is any four points but none three of them are colinear,

$\triangleright \quad$ there are no other affine simplexes in $\mathbb{R}^{2}$.

(ii) A set of points $\delta \subset \mathbb{R}^{3}$ is an affine

$\triangleright \quad 3$-element simplex iff $\delta$ is three colinear points,

$\triangleright \quad 4$-element simplex iff $\delta$ is any four coplanar points but none three of them are colinear,

$\triangleright \quad 5$-element simplex iff $\delta$ is any five points but none four are coplanar (and thus none three of them are colinear),

$\triangleright \quad$ there are no other affine simplexes in $\mathbb{R}^{3}$.

The connection between linear algebraic and affine simplexes will be discussed in Definition 6 and Proposition 2. From affine spaces we can move towards hypergraphs (set systems):

Definition 4. Let $(\mathcal{V}, \mathcal{E})$ be any hypergraph. Any edge (set of vertices) $E \in \mathscr{E}$ is called a hypergraph simplex if it is inclusion-minimal, that is $E$ does not contain any other edge $F \in \mathcal{E}$ :

$$
F \nsubseteq E \text { for } F \in \mathcal{E} \text {. }
$$

\section{APPLICATIONS}

One of the practical applications of linear algebraic simplexes is in stoichiometry when studying minimal reactions and mechanisms.

Consider for example the chemical reaction

$$
\mathrm{NaOH}+\mathrm{HNO}_{3}=\mathrm{NaNO}_{3}+\mathrm{H}_{2} \mathrm{O}
$$

which corresponds to the vector-equation

$$
[1,0,1,1]^{T}+[1,1,0,3]^{T}=[0,1,1,3]^{T}+[2,0,0,1]^{T}
$$


or

$$
[1,0,1,1]^{T}+[1,1,0,3]^{T}-[0,1,1,3]^{T}-[2,0,0,1]^{T}=[0,0,0,0]^{T}
$$

where the first coordinates correspond to (the number) of $H$ in each molecule, the second ones to $N$, the third ones to $N a$ and the fourth ones to $O$. In other words we could say that in $\mathbb{R}^{4}$ we use the (linear algebraic) "basis" $B=\{H, N, N a, O\}$ instead of the usual one $\left\{\underline{e}_{1}, \underline{e}_{2}, \underline{e}_{3}, \underline{e}_{4}\right\}$.

In general: let the chemical species ("molecules") $A_{1}, A_{2}, \ldots, A_{n}$ consist of chemical elements $E_{1}, E_{2}, \ldots, E_{m}$ as

$$
A_{j}=\sum_{i=1}^{m} a_{i, j} E_{i} \quad \text { where } a_{i, j} \in \mathbb{N} \text { for } j=1,2, \ldots, n .
$$

Write $\underline{A}_{j}$ for the vector $\left[a_{1, j}, a_{2, j}, \ldots, a_{m, j}\right]^{T}$. Using the law of mass-balance we know that there (might) exists a chemical reaction between the species $\left\{A_{j}: j \in S\right\}$ for any $S \subseteq\{1,2, \ldots, n\}$ if and only if the homogeneous linear equation

$$
\sum_{j \in S} x_{j} \underline{A}_{j}=\underline{0}
$$

has nontrivial solution for some $x_{j} \in \mathbb{R}, j \in S$, that is if the vector set $\left\{\underline{A}_{j}: j \in S\right\}$ is linearly dependent. (Each $x_{j}$ can be assumed an integer by (2.4).) The vectors $\underline{A}_{j}$ with positive coefficients $x_{j}>0$ in (2.5) denote the species the reaction starts with (on the left-hand-side of (2.1)), while the vectors $\underline{A}_{j}$ with negative coefficients $x_{j}<0$ in (2.5) denote the species the reaction ends with (on the right-hand-side). Of course the reactions obtained in the above way are only possibilities, e.g. the reaction

$$
2 \mathrm{Au}+6 \mathrm{HCl} \rightarrow 2 \mathrm{AuCl}_{3}+3 \mathrm{H}_{2}
$$

does not occur under normal conditions.

Considering a reaction like (2.1) or (2.6) one may ask whether the number of different kinds of molecules can be reduced, or, in other words, could react the remainding molecules if one of them is missing? Are the remainig vectors linearly dependent if one of (or more) them is/are missing? In the language of mathematics we can say:

Definition 5. The reaction in (2.5) is called a minimal reaction if for no $T \subsetneq S$ might there be any reaction among the species $\left\{A_{j}: j \in T\right\}$; that is if the vector set $\left\{\underline{A}_{j}: j \in T\right\}$ is linearly independent for any $T \subsetneq S$.

The above (chemical) notion explains the need of Definition 1 of linear algebraic simplexes. Indeed, the set of vectors, which correspond to molecules involved in a minimal reaction, form a (linear algebraic) simplex!

In our third example the species $A_{1}=\mathrm{C}, A_{2}=\mathrm{O}, A_{3}=\mathrm{CO}$ and $A_{4}=\mathrm{CO}_{2}$ determine the vectors $\underline{A}_{1}=[1,0], \underline{A}_{2}=[0,1], \underline{A}_{3}=[1,1]$ and $\underline{A}_{4}=[1,2]$. The vectorset $H=$ $\left\{\underline{A}_{1}, \underline{A}_{2}, \underline{A}_{3}, \underline{A}_{4}\right\}$ contains the simplexes

$$
\left\{\underline{A}_{1}, \underline{A}_{2}, \underline{A}_{3}\right\},\left\{\underline{A}_{1}, \underline{A}_{2}, \underline{A}_{4}\right\},\left\{\underline{A}_{1}, \underline{A}_{3}, \underline{A}_{4}\right\} \text { and }\left\{\underline{A}_{2}, \underline{A}_{3}, \underline{A}_{4}\right\} \text {. }
$$


After solving the corresponding equations (2.5), we have the following (complete) list of minimal reactions, corresponding to the above simplexes:

$$
\mathrm{C}+\mathrm{O}=\mathrm{CO}, \mathrm{C}+2 \mathrm{O}=\mathrm{CO}_{2}, \mathrm{O}+\mathrm{CO}=\mathrm{CO}_{2} \text { and } \mathrm{C}+\mathrm{CO}_{2}=2 \mathrm{CO} \text {. }
$$

In general, instead of considering (2.5) for some subsets $S$, we are invited to investigate the "full" homogeneous system of linear equations

$$
\sum_{j=1}^{n} x_{j} \underline{A}_{j}=\underline{0}, \quad \text { equivalently } \mathbf{A} \cdot \underline{X}=\underline{0}
$$

where

$$
\mathbf{A}:=\left[\underline{A_{1}}, \underline{A_{2}}, \ldots, \underline{A_{n}}\right] \in \mathbb{R}^{m \times n} \quad \text { and } \quad \underline{X}=\left[x_{1}, \ldots, x_{n}\right] \in \mathbb{R}^{n} .
$$

The solution vectors $\underline{X}$ of (2.8) are any (possible) reactions among the species $\left\{A_{1}, A_{2}, \ldots, A_{n}\right\}$.

Denote now the set of (all) solutions of (2.8) by $\mathbf{M}_{\mathbf{A}}$ :

$$
\mathbf{M}_{\mathbf{A}}:=\left\{\underline{X} \in \mathbb{R}^{n} \mid \mathbf{A} \cdot \underline{X}=\underline{0}\right\} .
$$

In practice, a chemical mechanism is a sequence, i.e. a linear combination of reactions (which correspond to solution-vectors $\underline{X} \in \mathbf{M}_{\mathbf{A}}$ ), so for any mechanism $\mathcal{M}$

$$
\mathcal{M}=\sum_{\ell=1}^{k} m_{\ell} \underline{X}_{\ell}
$$

for some vectors $\underline{X}_{1}, \ldots, \underline{X}_{\ell} \in \mathbf{M}_{\mathbf{A}}$ and for some $k \in \mathbb{N}$. Since any mechanism satisfies the law of mass-balance, we must have

$$
\mathbf{A} \cdot \mathcal{M}=\underline{0}
$$

which clearly follows from the definition (2.10) of $\mathbf{M}_{\mathbf{A}}$ as

$$
\mathbf{A} \cdot \mathcal{M}=\sum_{\ell=1}^{k} m_{\ell} \mathbf{A} \cdot \underline{X}_{\ell}=\underline{0}
$$

In this way we get the second step of the hierarchy: the vectors $\underline{X}_{\ell}$ of the equality (2.12) are, in fact, all the solutions of the previous equality (2.8). The final connection among the coefficients $\underline{A}_{j}$ of (2.8) and all of the solutions $M:=\left[m_{1}, \ldots, m_{\ell}\right]$ of (2.12) has been raised and planned to investigate in [13].

The structure of minimal solutions of homogeneous systems of linear equations with connections to simplexes are discussed in [22].

Linear algebraic simplexes can also be used for finding dimensionless groups in dimensional analysis: we investigate the structure of composite measure units in physics (see e.g. $[6,11,16]$ and $[14])$. 
Let there be given $m$ elementary quantities (mass,lenght,time,etc.) denoted by $E_{1}, \ldots, E_{m}$ and $n$ quantities $A_{1}, \ldots, A_{n}$ ( $n \in \mathbb{N}$ is any number) as

$$
A_{j}=\prod_{i=1}^{m} E_{i}^{a_{i, j}}
$$

where $a_{i, j} \in \mathbb{Z}$ for $j=1, \ldots, n$ and $i=1, \ldots, m$. Clearly we can assign the quantities $A_{j}$ to the vectors

$$
\mathbf{A}_{j}:=\left[a_{1, j}, \ldots, a_{m, j}\right]^{T} \in \mathbb{R}^{m}
$$

for $j=1, \ldots, n$, which vectors are in the $m$-dimensional linear space $\mathbb{R}^{m}$. Now, a (possible) dimensionless group (real number) of the quantities $\left\{A_{j}: j \in S\right\}$ does exists for any $S \subseteq\{1, \ldots, n\}$ iff the equality

$$
\prod_{j \in S} A_{j}^{x_{j}}=1
$$

holds, or equivalently (considering the exponents) the homogeneous system of linear equations

$$
\sum_{j \in S} x_{j} \cdot A_{j}=\mathbf{0}
$$

has nontrivial solution for $x_{j} \in \mathbb{R}(j \in S)$. That is, we again reached to the system of linear homogen equations and to the problem of finding simplexes!

For example, consider the flow of a fluid through a heated tube and the heat transfer between the pipe wall and the fluid. Consider now the following quantities, which we also write in vectors form in the base $\{m, \ell, t, T\}$ :

$$
\begin{array}{lll}
A_{1}=d(\ell) & \text { tube diameter } & \mathbf{A}_{1}=[0,1,0,0] \\
A_{2}=v(s / t) & \text { linear velocity } & \mathbf{A}_{2}=[0,1,-1,0] \\
A_{3}=\rho\left(m / \ell^{3}\right) & \text { fluid density } & \mathbf{A}_{3}=[1,-3,0,0] \\
A_{4}=v(m / \ell t) & \text { viscosity } & \mathbf{A}_{4}=[1,-1,-1,0] \\
A_{5}=\kappa\left(A / t^{2} T\right) & \text { heat capacity } & \mathbf{A}_{5}=[0,2,-2,-1] \\
A_{6}=\lambda\left(m / t^{3} T\right) & \text { heat transfer coeff. } & \mathbf{A}_{6}=[1,0,-3,-1] \\
A_{7}=\mu\left(m \ell / t^{3} T\right) & \text { thermal conductivity } & \mathbf{A}_{7}=[1,1,-3,-1]
\end{array}
$$

For example, one minimal dimensionless group $X_{1}$ of the seven ones is the following:

$$
X_{1}=[0,0,0,1,1,0,-1]^{T}
$$

which corresponds to the equality

$$
v \cdot \kappa=\mu \cdot c
$$

for some constant $c \in \mathbb{R}$. 


\section{BASIC PROPERTIES}

For investigating the common properties of the different Defintions 1 through 4 , we denote the ground set with $\mathcal{R}$ in each case.

The following fundamental property is valid for all of these definitions.

Proposition 1. (i) For any set $X \subset \mathcal{R}$ either $X$ is a simplex itself, or $X$ strictly contains a simplex $Y \varsubsetneqq X$ or $X$ is strictly contained in a simplex $X \varsubsetneqq Z$. Exactly one of the previous three cases yields for all subset $X \subset \mathcal{R}$.

(ii) (follows from (i)) The simplexes in $\mathcal{R}$ form a Sperner system, i.e. for each two simplexes $S_{1}, S_{2} \subset \mathcal{R}\left(S_{1} \neq S_{2}\right)$ we have

$$
S_{1} \nsubseteq S_{2} \text { and } S_{1} \nsubseteq S_{2} .
$$

The main problem of our investigations in the last decade was the following:

Problem 1. For given natural numbers $m, n \in \mathbb{N}$ what is the minimal and maximal possible value of the number of simplexes $8 \subset \mathscr{H}$ if $\mathscr{H}$ can be any set $\mathscr{H} \subset \mathbb{R}^{n}$ satisfying that $\mathscr{H}$ spans $\mathbb{R}^{n}$ and $|\mathcal{H}|=m$, and what are the extremal sets $\mathscr{H}$ (having least/maximal number of simplexes $\delta \subset \mathscr{H}$ )?

This problem can be raised for any kind of simplexes.

For technical simplifications we introduce

Notation 1 . For any finite set $\mathscr{H} \subset \mathbb{R}^{n}$ we denote by $\operatorname{simp}_{\ell}(\mathscr{H})$ the number of linear algebraic and by $\operatorname{simp}_{a}(\mathscr{H})$ the number of affine simplexes $\delta \subset \mathscr{H}$ contained in $\mathscr{H}$. For $2 \leq k \leq n+1$ we denote by $\operatorname{simp}_{k}(\mathscr{H})$ the number of $k$-element (linear algebraic) simplexes in $\mathscr{H}$.

The following assumption (which is originally not supposed in Problem 1), plays a crucial role in later investigations:

Condition 1 . $\mathscr{H}$ contains no parallel vectors, especially $\underline{0} \notin \mathscr{H}$.

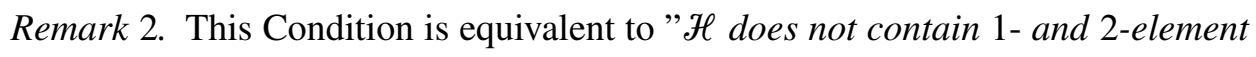
linear algebraic simplexes" (see Remark 1).

Recall, that parallel vectors in $\mathscr{H}$ represent isomers or different doses of molecules, etc.

In the shade of the above Condition we can replace each vector by its scalar multiplier and reduce the dimension as follows.

Definition 6. For any $\underline{h} \in \mathbb{R}^{n}$ we let

$$
\Lambda \underline{h}:=\{\lambda \cdot \underline{h}: \lambda \in \mathbb{R}, \lambda \neq 0\}
$$

and for $\mathscr{H} \subset \mathbb{R}^{n}$

$$
\Lambda \mathscr{H}:=\{\Lambda \underline{h}: \underline{h} \in \mathscr{H}\}
$$


and

$$
\mathscr{H}^{\mathcal{P}}:=\Lambda \mathscr{H} \cap \mathcal{P}
$$

for an $n-1$-dimensional affine hyperplane $\mathcal{P} \subset \mathbb{R}^{n}$ which is not parallel to any $\underline{h} \in \mathscr{H}$.

(For finite or at most countable set $\mathscr{H}$ such a $\mathcal{P}$ always exists.)

Proposition 2. There is a bijective correspondence between $\mathscr{H}^{\mathcal{P}} \subset \mathcal{P}$ (i.e. $\mathscr{H}^{\mathcal{P}} \subset$ $\left.\mathbb{R}^{n-1}\right)$ and $\mathscr{H} \subset \mathbb{R}^{n}$, and similarly between $\operatorname{simp}_{a}\left(\mathscr{H}^{\mathcal{P}}\right)$ and $\operatorname{simp}_{\ell}(\mathscr{H})$, so

$$
\left|\mathscr{H}^{\mathcal{P}}\right|=|\mathscr{H}| \quad \text { and } \operatorname{simp}_{a}\left(\mathscr{H}^{\mathcal{P}}\right)=\operatorname{simp}_{\ell}(\mathscr{H}) .
$$

(Actually we map $\mathscr{H}$ into the projective space $P\left(\mathbb{R}^{n}\right)$. It is not too hard to see, that the sets $\mathcal{S}^{\mathcal{P}} \subset \mathcal{P}$ form affine simplexes (in the sense of Definition 3 ) for each linear algebraic simplex $8 \subseteq \mathscr{H}$. The cases $n=3$ and $n=4$ in Definition 1 are clear 2 and 3 -dimensional concepts.

The other notions of simplexes (in matroids and in hypergraphs) are pure mathematical generalizations only at this moment.

\section{ON THE NUMBER OF SIMPLEXES}

First we present some results concerning Problem 1.

In [17] and [19] a polynomial algorithm for listing all simplexes, contained in a given arbitrary finite set $\mathscr{H} \subset \mathcal{R}$, and related questions were discussed. Though only linear algebraic simplexes are mentioned in those papers, the algorithm works for any kind of set system, satisfying (i) of Proposition 1, too. The ground idea of our algorithm was to modify (in- or decrease) the actualy tested set (whether or not it is a simplex) to search for all simplexes in $\mathscr{H}$. Another approaches to and applications of the algorithmic problems can be found eg. in $[1,2,4,10]$.

We have the following theoretical results. First, in Theorems 1 through 4 we deal with linear algebraic simplexes, we list the results on other kinds of simplexes after.

Theorem 1 ([7]). For any $\mathscr{H} \subseteq \mathbb{R}^{n}$ of fixed size, such that $\mathscr{H}$ spans $\mathbb{R}^{n}, \operatorname{simp}(\mathscr{H})$ is maximal if and only if any $n$ vectors of $\mathscr{H}$ are linearly independent.

Remark 3. Such sets $\mathscr{H}$ of any size can be easily constructed using the property of Vandermonde determinants: $\mathscr{H}=\left\{h_{1}, \ldots, h_{m}\right\}$ where $h_{i}=\left[1, i, i^{2}, \ldots, i^{n-1}\right]^{T} \quad(i \leq$ $m=|\mathscr{H}|)$.

Corollary 1. For any $\mathscr{H} \subseteq \mathbb{R}^{n},|\mathscr{H}|=m$ and $\mathscr{H}$ spans $\mathbb{R}^{n}$ we have

$$
\operatorname{simp}(\mathscr{H}) \leq\left(\begin{array}{c}
m \\
n+1
\end{array}\right)
$$

and equality holds if and only if any $n$ vectors of $\mathscr{H}$ are linearly independent. 
Though for each simplex $\delta \subseteq \mathscr{H}$ in $\mathbb{R}^{n}$ we have $|\delta| \leq n+1$ and by Proposition 1.(ii) the set of simplexes forms a Sperner system, Theorem 1 and Corollary 1 are not direct consequences of Sperner's well known theorem, since we have to ensure that each $(n+1)$-element subset of $\mathscr{H}$ form a simplex. Moreover, our specific (short) proof reveals the inner structure of simplexes and the reason for the uniquess of the maximal construction.

The other direction is much harder.

Theorem 2 ([7]). For any $\mathscr{H} \subseteq \mathbb{R}^{n}$ of fixed size such that $\mathscr{H}$ spans $\mathbb{R}^{n}$, $\operatorname{simp}(\mathscr{H})$ is minimal if and only if $\mathcal{H}$ consists of $n$ collections of parallel vectors of sizes differing by at most one from each other.

The proofs of Theorems 1 and 2 run by modifying the vectors in $\mathscr{H}$ to increase $\operatorname{simp}(\mathscr{H})$ as long as possible. Again, this construction works for other kinds of simplexes, too, e.g. for matroids in Theorems 5 through 11.

Corollary 2. For any $\mathscr{H} \subseteq \mathbb{R}^{n}$, if $\mathscr{H}$ spans $\mathbb{R}^{n}$ and $|\mathcal{H}|=m$ where $m=a \cdot n+b$, $0<b<n$ we have

$$
b \cdot\left(\begin{array}{c}
a+1 \\
2
\end{array}\right)+(n-b) \cdot\left(\begin{array}{l}
a \\
2
\end{array}\right) \leq \operatorname{simp}(\mathscr{H}) .
$$

In the special case when $m$ is divisible by $n$ we can write

$$
n \cdot\left(\begin{array}{l}
\frac{m}{n} \\
2
\end{array}\right) \leq \operatorname{simp}(\mathscr{H}) .
$$

Let us emphasize that $\mathscr{H}$ above may contain parallel vectors, moreover the minimal construction is built up from as many parallel vectors as possible.

In the forthcoming research we investigated the case when $\mathscr{H}$ may not contain parallel vectors.

Our first result is in dimension $n=3$.

Theorem 3 ([8]). For any $\mathscr{H} \subseteq \mathbb{R}^{3}$ of fixed size not equal to 3, 4 or 7 , such that $\mathscr{H}$ spans $\mathbb{R}^{3}$ and no parallel vectors are allowed in $\mathcal{H}, \operatorname{simp}(\mathscr{H})$ is minimal if and only if $\mathcal{H}$ is contained in two intersecting planes (their intersection vector belongs to $\mathscr{H})$, one of which is of size 3. In other words: precisely when $\mathcal{H}$ contains 3 linearly independent vectors $\left\{u_{1}, u_{2}, u_{3}\right\}$, another vector $v$ colinear with $u_{1}$ and $u_{2}$ and the rest $\mathscr{H} \backslash\left\{u_{1}, u_{2}, u_{3}, v\right\}$ colinear with $u_{2}$ and $u_{3}$.

Corollary 3. For any $\mathscr{H} \subseteq \mathbb{R}^{3}$ such that $\mathscr{H}$ spans $\mathbb{R}^{3}$, no parallel vectors are allowed in $\mathscr{H}$ and $|\mathscr{H}|=m \geq 4$ we have

$$
\left(\begin{array}{c}
m-2 \\
3
\end{array}\right)+1+\left(\begin{array}{c}
m-3 \\
2
\end{array}\right) \leq \operatorname{simp}(\mathscr{H})
$$

and equality holds just in the case described in the previous Theorem 3. 
In the proof we used the technique described in Definition 6 for reducing the dimension from $\mathscr{H} \subseteq \mathbb{R}^{3}$ to $\mathscr{H}^{\mathcal{P}} \subset \mathbb{R}^{2}$, in the meanwhile we transformed linear algebraic simplexes into affine ones (see Definition1). The minimal configurations for $|\mathcal{H}|=3,4,7$ are almost completely described in [8], P.Sellers drew our attention to the missing one in a personal communication.

For the general problem for minimizing $\operatorname{simp}(\mathscr{H})$ when $\mathscr{H}$ spans $\mathbb{R}^{n}$ and no parallel vectors allowed in $\mathscr{H} \subset \mathbb{R}^{n}$ we have the below conjecture (thanks to student Jianzhong Meng for his help with some calculations with Maple).

Conjecture 1 ([8]). In the case of $\mathscr{H} \subset \mathbb{R}^{n}, \mathscr{H}$ spans $\mathbb{R}^{n}$ and no parallel vectors are allowed in $\mathscr{H}$, the minimum for $\operatorname{sim} p(\mathscr{H})$ is attained precisely for the following configurations:

(i) if $n$ is even, then $\mathscr{H}$ contains $n$ linearly independent vectors $\left\{u_{i}: i=1, \ldots, n\right\}$ and the remaining divided as evenly as possible between the planes $\left\{\left[u_{i}, u_{i+1}\right] ; i=\right.$ $1,3, \ldots, n-1\}$,

(ii) if $n$ is odd, then $\mathscr{H}$ again contains $n$ linearly independent vectors $\left\{u_{i}: i=\right.$ $1, \ldots, n\}$, one special vector in the plane $\left[u_{n-1}, u_{n}\right]$ and finally the remaining vectors divided as evenly as possible between the planes $\left\{\left[u_{i}, u_{i+1}\right] ; i=1,3, \ldots, n-2\right\}$ with lower indices having precedence.

Our latest result is for the dimension $n=4$, we again used the technique described in Definition 6 for reducing the dimension from $\mathscr{H} \subseteq \mathbb{R}^{4}$ to $\mathscr{H}^{\mathcal{P}} \subset \mathbb{R}^{3}$, dealing with affine simplexes in $\mathbb{R}^{3}$ :

Theorem 4 ([15]). Assuming $\mathscr{H} \subset \mathbb{R}^{4}$, no parallel vectors allowed in $\mathscr{H}$ but $\mathscr{H}$ spans $\mathbb{R}^{4}$ (i.e. $\mathscr{H}^{\mathcal{P}} \subset \mathbb{R}^{3}$ is not coplanar) and $|\mathcal{H}|=m \geq 24$ we have

$$
\left(\begin{array}{c}
\lfloor m / 2\rfloor \\
3
\end{array}\right)+\left(\begin{array}{c}
\lceil m / 2\rceil \\
3
\end{array}\right) \leq \operatorname{simp}(\mathscr{H})
$$

and equality holds just in the case if $\mathscr{H}^{\mathcal{P}}$ is contained in two skew (detour) lines and these lines contain $\lfloor m / 2\rfloor$ and $\lceil m / 2\rceil$ many points of $\mathscr{H}^{\mathcal{P}}$.

The general problem for $\mathscr{H} \subset \mathbb{R}^{n}$ assuming that $\mathscr{H}$ spans $\mathbb{R}^{n}$ and no parallel vectors are in $\mathscr{H}$, still remains open.

For matroids we have the following results and open problem:

Theorem 5 ([3]). If $m>n+1$, then only the uniform matroid $U_{m, n}$ contains the maximum number of circuits, $\left(\begin{array}{c}n+1 \\ m\end{array}\right)$. If $m=n+1$, all matroids of size $m$ and of rank $n$ contain exactly 1 circuit.

Theorem 6 ([3]). Only the uniform matroid $U_{m, n}$ contains the maximum number of bases, namely $\left(\begin{array}{c}m \\ n\end{array}\right)$.

Theorem 7 ([3]). For each $m$ and $n$, there is a unique matroid $M_{o}$ of size $m$ and of rank $n$ containing the minimum number of bases, namely 1 , when we allow loops in the matroid. 
Theorem 8 ([3]). Any matroid $M$ of size $m$ and of rank $n$ contains at least $n-m$ circuits. A matroid contains exactly $m-n$ circuits if and only if the circuits of the matroid are pairwise disjoint.

Remark 4 . The matroid $M_{o}$ mentioned above also contains exactly $m-n$ pairwise disjoint circuits, i.e. loops.

Theorem 9 ([3]). Suppose that there are no large circuits and no loops in the matroid $M$, and let $\left\{a_{1}, a_{2}, \ldots, a_{n}\right\}$ be any fixed base. If $\vartheta_{i}$ denotes the number of elements in $M$ parallel to $a_{i}$ (including $a_{i}$ itself) for $i=1,2, \ldots, n$, then $M$ contains the minimum number of circuits iff $\left|\vartheta_{i}-\vartheta_{j}\right| \leq 1$ for $i \neq j$.

Corollary 4 ([3]). The minimum number of circuits in a matroid of size $m$ and of rank $n$, where $m=a n+b(0 \leq b<n)$, is

$$
b \cdot\left(\begin{array}{c}
a+1 \\
2
\end{array}\right)+(n-b) \cdot\left(\begin{array}{l}
a \\
2
\end{array}\right)
$$

and in particular, if $m$ is a multiple of $n$ :

$$
n \cdot\left(\begin{array}{c}
\frac{m}{n} \\
2
\end{array}\right) .
$$

Theorem 10 ([3]). a) For $m<2 n$, a matroid of size $m$ and rank $n$ contains the minimum number of circuits iff all its circuits are disjoint.

b) For $m \geq 2 n$, a matroid contains the minimum number of circuits iff it contains only 2-element circuits (i.e. parallel elements), and the sizes of the equivalence classes of parallel elements differ by at most 1 .

Remark 5. (i) There are many matroids satisfying a) while the matroids described in b) are, in fact, isomorphic.

(ii) The last part of the above proof describes uniquely the structures of matroids containing the minimum number of circuits when $m \geq 2 n$.

Theorem 11 ([3]). A matroid $M$ of size $m$ and rank $n$ contains the minimum number of bases iff it has a base $\left\{a_{1}, a_{2}, \ldots, a_{n}\right\}$ such that all other elements in $M$ are parallel to $a_{1}$.

Corollary 5 ([3]). The minimum number of bases is $m-n+1$, and the minimum configuration is unique.

Problem 2 ([3], still open). Characterize the matroids with the minimum number of circuits and bases, when neither parallel elements nor loops are allowed.

Our results concerning hypergraphs are planned to be published in [21].

Geometric simplexes and their chemical applications are discussed e.g. in [5,9, 18]. 
The strong connection between minimal reactions and minimal solutions of the homogeneous system of linear equations (2.5) and (2.8) was studied first in [12] and extended in [22].

In [20] the related notion of valuation operators is discussed in detail.

\section{ACKNOWLEDGEMENT}

Many thanks to the referee for valuable comments.

\section{REFERENCES}

[1] B. Bertók, L. T. Fan, and F. Friedler, "A graph-theoretic method to identify candidate mechanisms for deriving the rate law of a catalytic reaction," Computers and Chemistry, vol. 26, pp. 265-292, 2002.

[2] B. Bertók, L. T. Fan, F. Friedler, and S. Shafie, "Mechanisms of ammonia-synthesis reaction revisited with the aid of a novel graph-theoretic method for determining candidate mechanisms in deriving the rate law of a catalytic reaction," Hung.J. Ind. Chem., vol. 29, pp. 71-80, 2001.

[3] G. Dósa, I. Szalkai, and C. Laflamme, "The maximum and minimum number of circuits and bases of matroids," PU.M.A., Pure Math. Appl., vol. 15, no. 4, pp. 383-392, 2004.

[4] U. Haus, R. Hemmecke, and S. Pokutta, "Reconstructing biochemical cluster networks," Arxiv preprint, 2009.

[5] M. Hujter, "On the definition of the $n$-dimensional regular simplexes using inequalities," BME, Tech. Rep., 2009.

[6] S. Kumar and A. Pethô, "Note on a combinatorial problem for the stoichiometry of chemical reactions," Intern. Chem. Eng., vol. 25, pp. 767-769, 1985.

[7] C. Laflamme and I. Szalkai, "Counting simplexes in $\mathbb{R}^{n}$," Hung. J. Ind. Chem., vol. 23, pp. 237240, 1995.

[8] C. Laflamme and I. Szalkai, “Counting simplexes in $\mathbb{R}^{3}$," Electron. J. Comb., vol. 5, p. 11, 1998.

[9] P. Marcand, L. Lefebvre, L. Courvoisier, G. Perez, J. Counioux, and G. Coquerel, "Discontinuous isoperibolic thermal analysis (DITA) applied to organic components," J. Physique, vol. IV, no. 11, pp. 115-122, 2001.

[10] D. Papp and B. Vizvári, "Effective solution of linear Diophantine equation systems with an application in chemistry," J. Math. Chem., vol. 39, no. 1, pp. 15-31, 2006.

[11] A. Pethő, Lectures on linear algebraic methods in chemical engineering mathematics (stoichiometry). Univ. Hannover, Germany: Institut für Technische Chemie, 1990.

[12] A. Pethö, "On a class of solutions of algebraic homogeneous linear equations," Acta Math. Acad. Sci. Hung., vol. 18, pp. 19-23, 1967.

[13] P. Sellers, B. Szalkai, and I. Szalkai, "On linear algebraic foundations of reaction mechanisms," in preparation.

[14] P. H. Sellers, "Torsion in biochemical reaction networks," J. Math. Chem., vol. 47, no. 4, pp. 1287-1302, 2010.

[15] B. Szalkai and I. Szalkai, "Counting minimal reactions with specific conditions in $\mathbb{R}^{4}$," J. Math. Chem., vol. 49, no. 5, pp. 1071-1085, 2011.

[16] I. Szalkai, "Generating minimal reactions in stoichiometry using linear algebra," Hung. J. Ind. Chem., vol. 19, pp. 289-292, 1991.

[17] I. Szalkai, "Generating minimal reactions in stoichiometry using linear algebra," Hung. J. Ind. Chem., vol. 19, pp. 289-292, 1991.

[18] I. Szalkai, "Handling multicomponent systems in $\mathbb{R}^{n}$, I.: Theoretical results," J. of Math. Chem., vol. 25, pp. 31-46, 1999. 
[19] I. Szalkai, "A new general algorithmic method in reaction syntheses using linear algebra," J. of Math. Chem., vol. 28, pp. 1-34, 2000.

[20] I. Szalkai, "On valuation operators in stoichiometry and in reaction syntheses," J. of Math. Chem., vol. 27, pp. 377-386, 2000.

[21] I. Szalkai and Z. Tuza, "On minimal number of semi-simplexes in hypergraphs," in preparation.

[22] I. Szalkai, G. Dósa, Z. Tuza, and B. Szalkai, "On minimal solutions of systems of linear equations with applications,” Math. Notes, Miskolc, vol. 13, no. 2, pp. 529-541, 2012.

Authors' addresses

Balázs Szalkai

MSc student in Mathematics, Eötvös Lóránd University, Budapest, Hungary

E-mail address: bszalkaiegmail.com

\section{István Szalkai}

Department of Mathematics, University of Pannonia, POB. 158., H-8201 Veszprém, Hungary

E-mail address: szalkai@almos. uni-pannon. hu 